
ТЕЧЕНИЕ СИСТЕМНОГО ОСТЕОПОРОЗА

\author{
(․ С.С. Родионова, Л.Ю. Дарчия, У.Р. Хакимов
}

ФГБУ «Центральный институт травматологии и ортопедии им. Н.Н. Приорова» Минздрава России, Москва, Россия

Представлен обзор литературы о механизмах острой и хронической боли при переломах тел позвонков, осложняющих течение остеопороза. Приведены данные фармакологических и нефармакологических методов купирования боли при переломах тел позвонков, которые предшествуют назначению патогенетической терапии остеопороз или сочетаются с ней.

кЛЮчЕВЫЕ СЛОВА: Остеопороз; перелом тел позвонков; острая боль; хроническая боль; ортезы;

\title{
ACUTE AND CHRONIC PAIN IN VERTEBRAL FRACTURES AS SYSTEMIC OSTEOPOROSIS COMPLICATION
}

\author{
(c) S.S. Rodionova, L.U. Darchia, U.R. Khakimov
}

N.N. Priorov Central Scientific Research Institute of Traumatology and Orthopedics, Moscow, Russia

A literature review is devoted to acute and chronic pain mechanisms in vertebral fractures complicating osteoporosis. The data of pharmacological and non-pharmacological methods of pain relief for vertebral bodies fractures, which precede the pathogenetic therapy of osteoporosis or could be combined with it.

KEYWORDS: Osteoporosis; vertebral fractures; acute pain; chronic pain; orthoses;

Переломы тел позвонков относят к «переломам-маркерам» остеопороза, но из-за бессимптомного течения, [1] три четверти пациентов не обращаются за медицинской помощью, а возникающие в последующем болевые симптомы объясняют наличием дегенеративных изменений в позвоночнике. В то же время, эти болевые ощущения обусловлены, прежде всего переломом, так как его наличие ведет к дополнительной перегрузке фасеточного сустава и как следствие-перерастяжению капсульно - связочного аппарата сустава и развитию дегенеративного артрита. Боль при переломе тела позвонка, в отличие от остеохондроза, появляется только при статической нагрузке, что связывают с реакцией чувствительных нервных волокон на микропереломы трабекул, число которых увеличивается на фоне дефицита МПК и снижения прочности оставшихся костных структур в компримированном позвонке. В то время как плотность чувствительных нервных волокон в ткани, не снижается [2].

В случаях, когда пациенты связывают боль в позвоночнике с возникшим переломом тела позвонка, она появляется внезапно среди полного здоровья и пациенты могут указать на обстоятельства, предшествующие появлению боли. Чаще всего это подъем тяжести, иногда просто наклон туловища или резкий поворот, нередко указания на травмирующий момент отсутствуют. Боль в этих случаях ощущается на уровне поврежденного позвонка и, при локализации в грудном отделе, может носить опоясывающий характер [3], что требует проведения дифференциальной диагностики с инфарктом миокарда, плевритом, острым заболеванием органов брюшной полости. Острая боль при переломе тела позвонка обусловлена периостальным кровоизлиянием, чрезмерным количеством одновременно возникших микропереломов трабекул, чрезмерным натяжением связок вследствие этих изменений, спазмом параспинальных мышц. Отсутствие лечения остеопороза в таких случаях не только увеличивает риск новых переломов тел позвонков, но и способствует формированию хронической боли, которая является следствием механического сдавления связок и мышц из-за кифоза грудного отдела позвоночника, чрезмерного напряжения связок за счет снижения роста и развития артритов межпозвонковых суставов, укорочения и нарастающего спазма параспинальных мышц из-за снижения роста [3]. Некоторые пациенты при множественных переломах тел позвонков указывают на боль «во всех костях», которая появляется при любых сотрясениях [4]. Особенностью хронической боли при переломах тел позвонков является ее «интермиттирующий» характер: через 3-4 месяца ее интенсивность даже без лечения снижается, но затем неизбежное следует обострение, так как деформация позвоночника прогрессирует: усиливается грудной кифоз, формируется или, наоборот, выпрямляется гиперлордоз поясничного отдела позвоночника; из-за кифоза грудного отдела развивается гиперлордоз шейного отдела позвоночника, с напряжением мышц шеи и затылочной болью. В последующем из-за укорочения торса и давления ребер на гребни подвздошных костей (реберные дуги «садятся» на крылья тазовых костей) образуются выраженные кожные складки на боковой поверхности грудной клетки и появляются боли в боковых отделах туловища [5]. Из-за перерастяжения капсульно-связочного аппарата фасеточных суставов локализация боли не всегда соответствует уровню перелома [6]. Грудной кифоз, как следствие переломов тел позвонков грудного отдела позвоночника, формируется постепенно и сопровождается нарастающей слабостью мышц межлопаточной и паравертебральной области, что может стать причиной прогрессирования поясничного сколиоза и снижению роста на 10-15 см. Показателем уменьшения роста является появление разницы между расстояниями симфиз-стопа и голова-симфиз (в норме расстояния равны). 
При системном остеопорозе пальпация остистых отростков позвонков чаще безболезненна, также может быть отрицательной проба с осевой нагрузкой (надавливание на голову или надплечья), но сдавление грудной клетки может быть болезненным. Эти пробы должны проводиться осторожно во избежание новых переломов тел позвонков.

При переломах тел поясничных позвонков может появиться ограничение движений в поясничном отделе позвоночника, и пациенты пытаются поддерживать туловище в вертикальном положении с помощью рук или трости, особенно во время свежих болевых атак. Следует так же отметить изменение с возрастом восприятия и реагирования на боль, причина которого остается неясной. По мнению ряда исследователей, это может быть обусловлено повышением болевого порога у пожилых людей. [7] Отсутствие лечения остеопороза при уже случившемся переломе служит фактором дальнейшего прогрессирования заболевания и нарастания как симптоматики, так и новых переломов, поэтому всем пациентам старше 50 лет с указанием на выше приведенные жалобы или симптомы необходимо для уточнения диагноза остеопороза выполнить рентгенографию грудного и поясничного отделов позвоночника (уточнения степени компрессии тел позвонков) и рентгеновскую денситометрию для уточнения величины потери МПК [8]. После оценки гомеостаза кальция и маркеров ремоделирования необходимо начинать лечение остеопороза препаратами для коррекции нарушений костного ремоделирования (принципы назначения этих препаратов изложены в клинических рекомендациях Российской ассоциации по остеопорозу и Российской ассоциации эндокринологов) [9]. Однако на начальном этапе купирование боли и профилактика повторных переломов тел позвонков требует комплексного подхода, что сокращает время пребывания на постельном режиме, так как длительная разгрузка может стать фактором прогрессирования заболевания, ухудшения физического состояния, вызывает гипотрофию мышц, приводит к развитию пролежней, тромбозу глубоких вен, болезням органов дыхания, дезориентации и депрессии [10]. С этой целью используются различные обезболивающие препараты [11], разгрузка позвоночного столба с помощью ортезов [12], при отсутствии противопоказаний - физиотерапия [13, 14]. Длительность постельного режима при переломе тела позвонка на фоне остеопороза должна быть минимальной (не более 2-3 дней) и без ограничений, т.е. больным разрешается вставать в корсете на кратковременный срок $[15,16]$. Купирование боли в этот период кроме того достигается использованием короткого курса пероральных или инъекционных форм нестероидных противовоспалительных препаратов (НПВП), миорелаксантов центрального действия [17]. Последние снижают мышечный спазм и содействуют сну, улучшают кровоснабжение в спазмированных мышцах, прерывают порочный круг «спазм-боль-спазм», оказывают мембрано стабилизирующее действие и, соответственно, выраженный анальгезирующий и миорелаксирующий эффекты. Применение толперизона в комплексной терапии с НПВП позволяет снизить дозы последних, уменьшая риск побочных явлений [18]. Возможно использование местного обезболивания в виде пластырей с анестетиками и/или холодных компрессов (аппликации с димексидом), паравертебральных блокад области переломов местными анестетиками [11]. Вертикализация пациента с помощью корсетов рекомендуется со 2-3 дня после травмы. Основное действие корсета при переломах тел позвон- ков на фоне остеопороза-это повышение внутрибрюшного давления, что позволяет разгрузить тела позвонков и тем самым не только уменьшить боль, но и предотвратить риск новых переломов. Корсеты уменьшают болевой синдром благодаря ограничению подвижности пораженного отдела позвоночника, способствуя тем самым раннему восстановлению физической активности [19]. Основными критериями выбора корсета являются уровень перелома позвонков, степень компрессии, интенсивность болевого синдрома, общая активность пациента [20].

При переломах в поясничном отделе позвоночника рекомендуется пояснично - крестцовый корсет средней степени жесткости, при переломах грудного отдела позвоночника кроме того, можно рекомендовать грудопоясничный корсет Spinomed, который обеспечивает выпрямление позвоночного столба и уменьшение выраженности кифоза за счет активации разгибателей спины, также этот корсет способствует стимуляции работы околопозвоночных мышц через включение биологической обратной связи. (Рис 1). У пациентов с переломами тел позвонков на фоне остеопороза ношение корсета в течение всего дня не приводит к атрофии мышц при условии ежедневной ходьбы не менее 1,5-2 часов в день и ежедневной ЛФК в положении «лежа» и «сидя».

Также можно использовать опорные устройства на колесах с ручным тормозом. В этом случае благодаря выпрямлению спины и уменьшению нагрузки на позвоночник не только уменьшает боль при передвижении, но и снижает риск новых переломов [21]. Для уменьшения боли в боковых отделах туловища можно рекомендовать специальные мягкие ремни, уменьшающие соприкосновение костных структур [22].

У пациентов с переломами тел позвонков необходимо с первого дня назначать упражнения для нервно-мышечной стабилизации грудопоясничного отдела позвоночника, не требующих вертикализации (пассивные и активные упражнения с мобилизацией верхних и нижних конечностей и шейного отдела позвоночника). ЛФК снижает выраженность боли за счет повышения тонуса разгибателей и улучшения координации. Щадящие упражнения в положении «лежа» с подушкой под тазом помогают предотвратить прогрессирование деформации позвонка, восстановить осанку, и уменьшить болезненную кифотическую деформацию [23].

После вертикализации назначается ЛФК на укрепление грудных и межреберных мышц. У пациентов с переломами тел позвонков на фоне остеопороза чрезвычайно важно выработать стереотип движений с сохранением прямой осанки, чтобы уменьшить нагрузку на позвоночник и снизить риск последующих переломов. Исключается тяжелая работа, подъемы тяжести и наклоны вперед и в стороны [24]. Общими принципами упражнений при остеопорозе [25] являются:

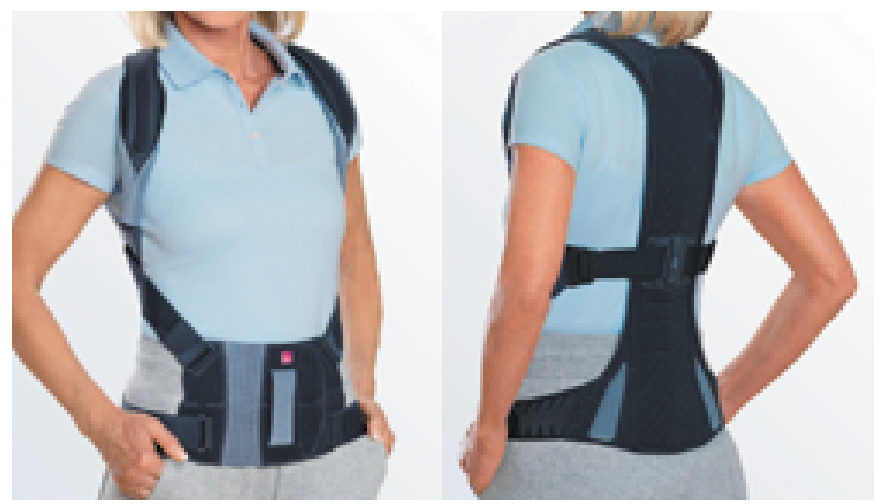

Рис. 1. Ортез «Spinomed IV» вид спереди и сзади. 
1. выборупражнений и интенсивность должны быть адаптированы к конкретному пациенту, чтобы избежать чрезмерного стресса позвоночника и вызывать новую травму

2. упражнения должны быть направлены на растяжение мышц и укрепление группы мышц, разгибающих позвоночник; ограничение упражнений со сгибанием и ротацией позвоночника;

3. упражнения выполняются осторожно в медленном темпе без резких движений;

4. при выполнении упражнений пациенты должны стараться выпячивать грудную клетку, глубоко дышать, выпрямлять спину и избегать кифотической позы;

5. важна регулярность упражнений (оптимально - ежедневно), так как после прекращения упражнений их положительный эффект быстро теряется.

Через 3 недели и в течение последующих 3 месяцев пациентам с переломами тел позвонков следует соблюдать режим «интермиттирующего» отдыха в горизонтальном положении»: после 2 ч пребывания в вертикальном положении необходимо 20 мин проводить в положении «лежа» $[26,27]$. Мануальная терапия категорически запрещена, но к настоящему времени выполнено одно исследование, которое предлагает мультимодальный подход, связывая щадящую мануальную терапию с физическими упражнениями. Авторы продемонстрировали эффективность программы в уменьшении боли и улучшения физической функции и выносливость мышц спины у пациентов с болевым синдромом на фоне перелома тел позвонков [28]. Однако эти рекомендации пока никто не подтвердил, осложнений в виде новых переломов тел позвонков после мануальной терапии много.

Лечение хронических болей при остеопорозе, осложненном переломами тел позвонков

Около 66 \% женщин, у которых остеопороз осложнился компрессионными переломами позвоночника, страдают от хронической боли в спине, при этом 26 \% испытывают ежедневную боль продолжительностью свыше 10 часов. Хронический болевой синдром связывают с неправильным или полным отсутствием лечения переломов тел позвонков в остром периоде [29]. В этом случае стимулом раздражения болевых рецепторов становится не только перелом трабекул, но их растяжение и сжатие. Усугубляется болевой синдром миофасциальными нарушениями, изменениями диско-теловых соотношений и развитием нарушений биомеханики вследствие деформаций позвоночного столба. Хроническая боль при переломах тел позвонков формирует у больных остеопорозом фобии.

Для купирования хронической боли рекомендуются курсы НПВП (при отсутствии противопоказаний со стороны желудочно-кишечного тракта) [30], но они оказывают негативное влияние на костный метаболизм, поэтому их использование не может быть длительным [31].
Анальгетики в виде аппликаций препаратов с димексидом (димексид 10\%-10,0 + новокаин 0,5\%-5,0 + димедрол 1\%-1,0 + вит-В12 250 мкг + диклонак 3.0) на область проекции боли на 4-6 часов 5-6 процедур; Рефлексотерапия (иглоукалывание, электропунктура, лазеропунктура). Для купирования миалгий рекомендуются курсы сирдалуда (2-4 мг 1-2 раза в день ,7-10 дней [32]. При патологических переломах позвонков на фоне остеопороза из-за сопутствующих заболеваний физиотерапия возможна лишь у 10-15\% пациентов [33]. Убедительных данных о возможности использования при остеопорозе для купирования хронической боли радиочастотной денервации медиальных ветвей дорсальных дуг, иннервирующих фасеточный сустав, нет.

Депрессия и нарушение сна у пациентов с хронической болью при переломах тел позвонков на фоне остеопороза требуют назначения антидепрессантов и помощи психолога. Для уменьшения психологических проблем, связанных с хроническим болевым синдромом, используют различные образовательные программы для пациентов с обучением самоконтролю [34]. Хроническую боль облегчает постоянное ношение корсетов (кроме сна) [12], которые разгружают позвоночник за счет восстановления «балонной» функции брюшной полости и повышая внутрибрюшное давление разгружают» позвоночник. Мягкие и полужесткие конструкции не вызывают атрофию мышечного «корсета»».

\section{ЗАКЛЮЧЕНИЕ}

Вероятность развития хронического болевого синдрома может быть снижена при своевременном назначении терапии, направленной на лечение остеопороза, целью которого прежде всего является предупреждение новых переломов и всех тех деформаций туловища, которые приводят к формированию болевого синдрома. Основополагающим в лечении патологических переломов тел позвонков, осложняющих течение остеопороза, является назначение патогенетических препаратов для лечения остеопороза (бисфосфонатов, деносумаба, терипаратида) с адекватным восполнением потребления солей кальция и купированием дефицита или недостаточности витамина D.

Пациенты с наличием длительного болевого синдрома не купируемого комплексным консервативным лечением могут быть кандидатами на такое малоинвазивное вмешательство как вертебропластика с последующей патогенетической терапией остеопороза, направленной прежде всего на предупреждение новых переломов костей скелета.

\section{ДОПОЛНИТЕЛЬНАЯ ИНФОРМАЦИЯ}

Конфликт интересов. Авторы декларируют отсутствие явных и потенциальных конфликтов интересов, связанных с публикацией настоящей статьи.

\section{СПИСОК ЛИТЕРАТУРЫ | REFERENCES}

1. Griffith JF. Identifying osteoporotic vertebral fracture. Quant Imaging Med Surg. 2015;5(4):592-602. doi: 10.3978/j.issn.2223-4292.2015.08.01

2. Jimenez-Andrade JM, Mantyh WG, Bloom AP, et al. The effect of aging on the density of the sensory nerve fiber innervation of bone and acute skeletal pain. Neurobiol Aging. 2012;33(5):921-932. doi: 10.1016/j.neurobiolaging.2010.08.008
3. Wu SS, Lachmann E, Nagler W. Current Medical, Rehabilitation, and Surgical Management of Vertebral Compression Fractures. J Women's Health. 2003;12(1):17-26. doi: 10.1089/154099903321154103

4. Sinaki $M$, Pfeifer $M$, Preisinger $E$, et al. The Role of Exercise in the Treatment of Osteoporosis. Current Osteoporosis Reports. 2010;8(3):138144. doi: 10.1007/s11914-010-0019-y 
5. Dreyfuss P, Tibiletti C, Dreyer SJ. Thoracic zygapophyseal joint pain patterns. A study in normal volunteers. Spine (Phila Pa 1976). 1994;19(7):807-811.

6. Francis RM, Aspray TJ, Hide G, et al. Back pain in osteoporotic vertebral fractures. Osteoporos Int. 2007;19(7):895-903. doi: 10.1007/s00198-007-0530-x

7. Gasik R, Styczyński T. Specifics of pharmacological treatment of back pains in people of old age. Polski Merkuliusz lekarski 2006; 21(124):394-397

8. Watts NB, Adler RA, Bilezikian JP, et al. Osteoporosis in Men: An Endocrine Society Clinical Practice Guideline. J Clin Endocr Metab. 2012;97(6):1802-1822. doi: 10.1210/jc.2011-3045

9. Мельниченко Г.А., Белая Ж.Е., Рожинская Л.Я., и др. Краткое изложение клинических рекомендаций по диагностике и лечению остеопороза российской ассоциации эндокринологов // Остеопороз и остеопатии. - 2016. - Т. 19. - №3. - С. 28-36. [Melnichenko GA, Belaya ZE, Rozhinskaya LY, et al. Summary of Clinical Guidelines for the Diagnosis and Treatment of Osteoporosis of the Russian Association of Endocrinologists. Osteoporosis and Bone Diseases. 2016;19(3):28-36. (In Russ.)] doi: 10.14341/osteo2016328-36

10. Chosa K, Naito A, Awai K. Newly developed compression fractures after percutaneous vertebroplasty: comparison with conservative treatment. Japanese Journal of Radiology. 2011;29(5):335-341. doi: 10.1007/s1 1604-011-0564-z

11. Ensrud KE, Schousboe JT. Vertebral Fractures. N Eng/ J Med. 2011;364(17):1634-1642. doi: 10.1056/NEJMcp1009697

12. Longo UG, Loppini M, Denaro L, et al. Osteoporotic vertebral fractures: Current concepts of conservative care. Br Med Bull. 2011;102(1):171-189. doi: 10.1093/bmb/ldr048

13. Barker KL, Javaid MK, Newman M, et al. Physiotherapy Rehabilitation for Osteoporotic Vertebral Fracture (PROVE): study protocol for a randomised controlled trial. Trials. 2014;15(1):22. doi: 10.1186/1745-6215-15-22

14. Denaro V, Longo UG, Denaro L. Vertebroplasty versus conservative treatment for vertebral fractures. The Lancet. 2010;376(9758):2071. doi: 10.1016/s0140-6736(10)62289-1

15. Longo UG, Loppini M, Denaro L, et al. Conservative management of patients with an osteoporotic vertebral fracture: A review of the literature. The Bone \& Joint Journal. 2012;94-B(2):152-157. doi: 10.1302/0301-620x.94b2.26894

16. Vorlat P, Leirs G, Tajdar F, et al. Predictors of Recovery After Conservative Treatment of AO-Type A Thoracolumbar Spine Fractures Without Neurological Deficit. Spine. 2010:1. doi: 10.1097/BRS.0b013e3181cdb5fc

17. Park Y-S, Kim H-S. Prevention and Treatment of Multiple Osteoporotic Compression Fracture. Asian Spine Journal. 2014;8(3):382. doi: 10.4184/asj.2014.8.3.382

18. van Tulder M, Becker A, Bekkering T, et al. Chapter 3 European guidelines for the management of acute nonspecific low back pain in primary care. Eur Spine J. 2006;15(S2):s169-s191. doi: 10.1007/s00586-006-1071-2

19. Rzewuska M, Ferreira M, McLachlan AJ, et al. The efficacy of conservative treatment of osteoporotic compression fractures on acute pain relief: a systematic review with meta-analysis. Eur Spine J. 2015;24(4):702-714. doi: 10.1007/s00586-015-3821-5
20. Pfeifer M, Begerow B, Minne HW. Effects of a New Spinal Orthosis on Posture, Trunk Strength, and Quality of Life in Women with Postmenopausal Osteoporosis. Am J Phys Med Rehabil. 2004;83(3):177-186. doi: 10.1097/01.phm.0000113403.16617.93

21. Frost HM. Personal Experience in Managing Acute Compression Fractures, their Aftermath, and the Bone Pain Syndrome, in Osteoporosis. Osteoporos Int 1998:8(1):13-15. doi: 10.1007/s001980050042

22. Bonner Jr FJ, Sinaki M, Grabois M, et al. Health Professional's Guide to Rehabilitation of the Patient with Osteoporosis. Osteoporos Int. 2003;14(0):1-22. doi: 10.1007/s00198-002-1308-9

23. Burke TN, França FJR, Meneses SRFd, et al. Postural control in elderly women with osteoporosis: comparison of balance, strengthening and stretching exercises. A randomized controlled trial. Clin Rehabil. 2012;26(11):1021-1031. doi: 10.1177/0269215512442204

24. Yoo J-H, Moon S-H, Ha Y-C, et al. Osteoporotic Fracture: 2015 Position Statement of the Korean Society for Bone and Mineral Research. Journal of Bone Metabolism. 2015;22(4):175. doi: $10.11005 / \mathrm{jbm} .2015 .22 .4 .175$

25. Sinaki M. Yoga Spinal Flexion Positions and Vertebral Compression Fracture in Osteopenia or Osteoporosis of Spine: Case Series. Pain Practice. 2013;13(1):68-75. doi: 10.1111/j.1533-2500.2012.00545.x

26. Hongo M, Miyakoshi N, Shimada Y, Sinaki M. Association of spinal curve deformity and back extensor strength in elderly women with osteoporosis in Japan and the United States. Osteoporos Int 2011;23(3):1029-1034. doi: 10.1007/s00198-011-1624-z

27. Angın E, Erden Z, Can F. The effects of clinical pilates exercises on bone mineral density, physical performance and quality of life of women with postmenopausal osteoporosis. J Back Musculoskelet Rehabil. 2015;28(4):849-858. doi: 10.3233/bmr-150604

28. Bennell KL, Matthews B, Greig A, et al. Effects of an exercise and manual therapy program on physical impairments, function and quality-of-life in people with osteoporotic vertebral fracture: a randomised, single-blind controlled pilot trial. BMC Musculoskel Disord. 2010;11(1). doi: 10.1186/1471-2474-11-36

29. Bianchi M, Orsini M, Saraifoger S, et al. Health and Quality of Life Outcomes. 2005;3(1):78. doi: 10.1186/1477-7525-3-78

30. Bavry AA, Khaliq A, Gong Y, et al. Harmful Effects of NSAIDs among Patients with Hypertension and Coronary Artery Disease. The American Journal of Medicine. 2011;124(7):614-620. doi: 10.1016/j.amjmed.2011.02.025

31. Vestergaard $\mathrm{P}$, Hermann $\mathrm{P}$, Jensen JEB, et al. Effects of paracetamol, non-steroidal anti-inflammatory drugs, acetylsalicylic acid, and opioids on bone mineral density and risk of fracture: results of the Danish Osteoporosis Prevention Study (DOPS). Osteoporos Int. 2011;23(4):1255-1265. doi: 10.1007/s00198-011-1692-0

32. Vellucci R, Mediati RD, Ballerini G. Use of opioids for treatment of osteoporotic pain. Clinical Cases in Mineral and Bone Metabolism. 2014;11(3):173-176.

33. Huang LQ, He HC, He CQ, et al. Clinical update of pulsed electromagnetic fields on osteoporosis. Chin Med J (Engl). 2008;121(20):2095-2099

34. Eisman JA, Bogoch ER, Dell R, et al. Making the first fracture the last fracture: ASBMR task force report on secondary fracture prevention. $J$ Bone Miner Res. 2012;27(10):2039-2046. doi: 10.1002/jbmr.1698

\section{ИНФОРМАЦИЯ ОБ АВТОРАХ [AUTHORS INFO]}

Родионова Светлана Семеновна, д.м.Н., профессор, руководитель научно-клинического центра остеопороза [Svetlana S. Rodionova, MD, PhD, professor]; адрес: Москва, 127299, ул. Приорова 10 [address: Moscow, Priorov st., 10, 127299]; телефон: +7 (499) 940-97-47; eLibrary SPIN: 3529-8052; e-mail: rod06@inbox.ru.

Дарчия Леван Юрьевич, к.м.н., сотрудник научно-поликлинического отделения [Levan Yu. Darchia, MD, PhD].

Хакимов Умеджон Рауфович, аспирант научно-клинического центра остеопороза [Umedzhon R. Khakimov, MD, PhD student]; eLibrary SPIN: 5316-8676.

\section{ЦИТИРОВАТЬ:}

Рожинская Л.Я., Белая Ж.Е., Луценко А.С. Новые возможности лечения вторичного гиперпаратиреоза у пациентов с терминальной стадией хронической болезни почек, получающих заместительную почечную терапию гемодиализом. // Остеопороз и остеопатии. - 2017. — Т. 20. - №1. — С.28-31. doi: 10.14341/osteo2017122-25

\section{TO CITE THIS ARTICLE:}

Rozhinskaya LY, Belaya ZE, Lutsenko AS. Novel treatment options for secondary hyperparathyroidism in end-stage kidney disease patients on hemodialysis therapy. Osteoporosis and bone diseases. 2017;20(1):28-31. doi: 10.14341/osteo2017122-25 